(e-migrinter

e-Migrinter

$18 \mid 2019$

L'ethnographie en migration(s)

\title{
Par-delà les émotions et la raison. Les apports de l'auto-analyse comme méthode de compréhension de nos terrains
}

Aurore Vermylen

\section{(2) OpenEdition \\ Journals}

Édition électronique

URL : https://journals.openedition.org/e-migrinter/1781

DOI : 10.4000/e-migrinter.1781

ISSN : 1961-9685

Éditeur

UMR 7301 - Migrinter

Référence électronique

Aurore Vermylen, «Par-delà les émotions et la raison. Les apports de l'auto-analyse comme méthode de compréhension de nos terrains ", e-Migrinter [En ligne], 18 | 2019, mis en ligne le 11 septembre 2019, consulté le 20 mai 2021. URL : http://journals.openedition.org/e-migrinter/1781 ; DOI : https:// doi.org/10.4000/e-migrinter.1781

Ce document a été généré automatiquement le 20 mai 2021.

Tous droits réservés 


\title{
Par-delà les émotions et la raison. Les apports de l'auto-analyse comme méthode de compréhension de nos terrains
}

\author{
Aurore Vermylen
}

\section{NOTE DE L'AUTEUR}

Les séjours de recherche menant aux présents résultats ont bénéficié de soutiens financiers du F.R.S.-FNRS. Je tiens à remercier Pierre-Joseph Laurent, et surtout sa femme Danielle Bastien, pour les quelques échanges que nous avons eus et qui m'ont permis d'affiner ma réflexion. Je remercie également Johan Cornez, David Berliner et Laurie Daffe qui m'ont recommandé des lectures. Je remercie enfin Jacinthe Mazzocchetti, Bochra Manaï et Amandine Desille pour leurs relectures et leurs précieux conseils.

\section{Introduction}

1 Alors qu'un chimiste travaille avec des fioles et des produits, l'anthropologue travaille avec son corps (il se rend sur place), son conscient (il s'intéresse activement et prend des notes) et son inconscient (il est sujet à des choses dont il n'a pas conscience) pour faire science. L'anthropologue est donc réellement un «instrument humain» (Powdermaker, 1966, p. 19) au service de la compréhension de ses terrains. Selon Powdermaker (1966, p. 19) :

"It is important to accept that this human instrument is as much a product of biological, psychological, and social conditioning as are the people he studies. [...] Obviously, this personality is not formed in the field but has many years of conditioning behind it. These influence the anthropologist's choice of problems and of methods, even the choice of 
discipline itself. [...] As Everett Huges writes, 'It is doubtful whether one can become a good social reporter unless he has been able to look, in a reporting mood, at the social world in which he was reared. "

Devereux, dans De l'angoisse à la méthode (1967), avance l'idée que la psychanalyse est un outil pour faire science en anthropologie, et lui serait complémentaire. Le « complémentarisme » serait, pour lui, non pas une théorie, mais une généralisation méthodologique.

"J'utilise systématiquement les outils conceptuels de la psychanalyse, mais je les traite comme des outils et non comme des dieux domestiques [...]. Qu'ils survivent ou non n'a aucun intérêt à mes yeux » (Devereux in Xanthakou, 1995, p. 183)1.

2 Je pars également du postulat que les outils de la psychanalyse doivent indéniablement faire partie de la valise de l'anthropologue. Sans pour autant oublier qu'ils ne sont que des outils en vue d'arriver à une analyse anthropologique consciente de ses biais, de ses nœuds, et de ses compréhensions. Comment la démarche réflexive et la pratique de l'auto-analyse parviennent-elles à dépasser l'opposition classique entre raison et émotion ?² En quoi les émotions du chercheur peuvent-elles servir à une compréhension plus fine des réalités sociales observées et étudiées ? Dans une première partie, j'expliquerai comment j'ai tenté, au cours de ma thèse, de développer une anthropologie consciente de ses limites, en essayant de dépasser mes " angles morts » (des contre-transferts ?) par l'auto-analyse. Dans une deuxième partie, où je décris le «second rôle» assigné à l'anthropologue, je réfléchirai aux projections que les interlocuteurs peuvent avoir sur nous, étrangers attentifs et attentionnés à leurs réalités quotidiennes. Par le biais d'un exemple tragique, je montrerai en quoi avoir conscience de ces projections (ces transferts ?) permet de 1) mieux appréhender son terrain et 2) nuancer la réflexion d'une posture modeste et égalitaire entre l'ethnologue et ses interlocuteurs. Enfin, dans une troisième partie, où je décris l'idée de "l'absorption des ambiances", je montrerai en quoi écouter nos émotions qui surgissent sur/à la suite de nos terrains constitue une véritable donnée en soi à analyser au même titres que nos carnets de terrain.

\section{Dépasser les « angles morts » par l'auto-analyse}

Dans un article intitulé Les risques du métier. Engagements problématiques en sciences sociales, Daniel Cefaï et Valérie Amiraux (2002, p. 4) décrivent les enjeux de l'« engagement du soi » sur ses terrains.

« L'auto-analyse en ethnographie ou l'ego-histoire ont [...] réfléchi sur les 'liaisons dangereuses' entre un choix d'objet et une histoire de vie. [...] [D'une part,] l'investissement intellectuel serait la transfiguration d'un investissement existentiel [...] dont l'élucidation appelle un travail d'auto-analyse. [...] [D'autre part,] les résonances entre expériences traversées et objets d'enquête peuvent donner lieu à des processus d'abréaction ou d'anamnèse, incontrôlés après de longues phases de refoulement et de dénégation. Plutôt que de se laisser prendre par surprise, mieux vaut travailler sur soi et s'engager dans une interrogation socio-analytique, comme Jean-Claude Passeron et, sur le tard, Pierre Bourdieu, nous y invitent. Ce risque de prendre le tour d'une interrogation obsessionnelle, d'une phobie méthodologique ou d'une répétition névrotique, une fois analysé, se transforme en atout de compréhension. »

4 Face à cette volonté de travailler mon «engagement du soi » sur mes terrains, j'ai entamé à l'époque du début de ma thèse de doctorat (2014-2015) une «courte » 
analyse ${ }^{3}$. Le projet était double : politique d'une part, et méthodologique d'autre part. Politique, parce qu'il me semblait nécessaire, dans un souci éthique de ce qui m'apparaissait comme de la réciprocité (voir infra), de laisser entrer dans mon intimité un analyste alors que j'allais moi-même observer et analyser les intimités des autres. Je voyais mon analyste noircir ses feuilles de papiers de mon "cas d'étude », comme je pouvais moi-même noircir mes carnets de terrains en interrogeant mes interlocuteurs. Je me disais alors OK, je peux comprendre ce que ça fait d'avoir un anthropologue face à soi. J'avais accès à l'intimité de mes interlocuteurs, je ne pouvais légitimement pas refuser qu'une personne (ici entendue dans le sens de "personne extérieure [que je voyais alors comme étant] 'neutre'») ait accès à ma propre intimité. Méthodologique, parce qu'il me semblait impensable de vouloir étudier des questions anthropologiques sans être consciente de mon propre vécu, de mes propres nœuds de tension, et donc de mes propres biais d'analyse. Tout comme Magali Aloatti (2019), qui a eu recours à des "para-ethnographes " comme partenaires de création de connaissance, j'avais moimême recours à mon analyste pour étudier mes propres réactions face aux terrains, mais aussi pour déconstruire mes propres schèmes de pensée. De fait, il me semblait qu'un bon travail anthropologique s'accompagnait d'une véritable écoute de soi-même pour pouvoir dépasser ses propres dénis, éblouissements ou convictions ${ }^{4}$. Dans la lignée de la pensée postmoderne ou d'auteurs comme Dominique Pestre (2003), je voyais dans mes biais subjectifs des dangers pour une théorisation trop rapide de mes terrains.

Pour illustrer ce propos, je reprendrai ici un célèbre exemple d'une analyse scientifique biaisée du fait de la non-conscience de la nécessité d'une démarche auto-analytique. Thibault Le Texier (2018), dans un livre intitulé Histoire d'un mensonge. Enquête sur l'expérience de Stanford sur la prison, montre comment les résultats de l'expérience de Stanford étaient totalement biaisés et construits (au point où il annonce avoir " découvert le pot aux roses »). Philip Zimbardo ${ }^{5}$ avait pour ambition de démontrer, sur la base de son a priori personnel et ses positions politiques antérieures à l'étude, la violence du système carcéral. Il montrait que toute personne mise dans une position de gardien de prison devenait, de facto, violente. Les résultats avaient été diffusés dès le premier jour de l'expérience, au compte-goutte, sans même laisser le temps à la recherche de se dérouler. Depuis, celle-ci a fait le tour du monde, tant dans les manuels universitaires de psycho-sociologie que hors du milieu académique, avec notamment le célèbre film Das Experiment ${ }^{6}$. Mais, selon les analyses de Thibault Le Texier, de retour dans les archives de l'expérience, rien ne pouvait alors prouver la violence du système carcéral. Invité à parler de son livre sur France Culture, Thibault Le Texier défendait l'idée de faire de la «méta-recherche ", c'est-à-dire une recherche à la fois consciente de ses biais méthodologiques et sociologiques. Il postulait alors :

Je suis vraiment partisan de l'auto-analyse, c'est-à-dire que le chercheur essaye de comprendre pourquoi il fait ses enquêtes, pourquoi il choisit tel type d'outil, pourquoi il choisit tel type d'objet, et ça c'est pour moi un réquisit de la scientificité des sciences sociales. Et le militantisme, évidemment on ne va pas prétendre que personne n'a de croyance, que personne n'a de valeur, mais il faut peut-être essayer de désamorcer les effets de ce militantisme et essayer de neutraliser les effets potentiels, de voir que d'avoir tel type de conviction peut nous amener à introduire tel type de biais dans nos recherches. (Thibault Le Texier, 5 mai 2018, invité sur France Culture, émission «De la suite dans les idées, Fake science, retour critique d'expérience à Stanford »)

6 Sur la base de deux types d'exemples, l'un provenant de mon propre parcours familial et l'autre de mes expériences de terrain, je vais mettre en résonnance cette ambition de dépoussiérer mes subjectivités, sujettes à mes aveuglements, pour prendre conscience 
de certaines caractéristiques des réalités sociales dans lesquelles je mène ma recherche. Par ces exemples, j'exposerai quels ont été les dangers de ne pas être consciente de mes propres « angles morts » lors de la construction d'une pensée sur les camps de réfugiés ou sur la guerre dans la région des Grands Lacs.

Ce sont les récits de la Seconde Guerre mondiale, qui ont forgé l'identité de ma famille, la première raison qui m'a décidée à développer une anthropologie des camps de réfugiés et de la guerre. Ils étaient récurrents aux soupers, et je me sentais presque « chez moi » dans cette époque pourtant lointaine du début des années quarante. Ma grand-mère était adolescente lors de la guerre, qu'elle avait vue (selon ce qu'elle nous a transmis) comme un jeu. Dans un esprit d'autodérision, elle nous racontait un nombre incroyable d'anecdotes de comment elle avait réussi à "avoir» les Allemands (qui occupaient alors la Belgique), ici en cachant du beurre dans ses jupes, là en prétendant ne pas comprendre l'allemand pour écouter en cachette des informations précieuses sur des cousins ou oncles qui avaient été emprisonnés. Ces anecdotes me donnaient presque envie de prendre une machine à remonter le temps et vivre la guerre à ses côté. Mon grand-père, lui, était rentré dans la résistance et avait fini par se faire arrêter et emprisonner un an avant la fin de la guerre. Il avait donc passé douze mois dans sept camps de travail. De ce parcours de vie, j'ai reçu notamment des bribes de discours du type "héro", où, même enfermé, il continuait à réciter des vers de l'Iliade et de l'Odyssée d'Homère, sous prétexte que l'on peut enfermer un homme, mais jamais son esprit. Cette année avait tout de même été dangereuse pour lui, puisqu'il avait frôlé une dizaine de fois la mort (exécutions, etc.), et avait étonnamment souvent été sauvé par son côté "grande gueule». Surtout, il avait toujours survécu. En découle une volonté familiale d'être toujours fort, plus fort que la guerre. Si la vie dans un camp de travail n'abat pas un homme, ne lui fait pas perdre sa poésie, alors rien ne peut l'abattre.

Ces récits m'ont donné l'envie de faire la thèse que je fais. Ils m'ont aussi, inconsciemment, donné l'envie de parler de la guerre et des camps selon une perspective bien particulière : celle de toujours voir le bon côté de ces situations. Tant que je n'avais pas travaillé ce biais avec ma psychanalyste, j'étais tout bonnement incapable de réaliser, de me rendre compte, que la vie dans les camps de réfugiés pour mes interlocuteurs pouvait être parfaitement horrible, difficile, voire affaiblissante. Je ne le voyais pas. Ce n'est que très récemment, en faisant mes derniers terrains dans des camps et auprès de réfugiés réinstallés ${ }^{7}$ mais y ayant vécu, que cela m'a sauté aux yeux : problèmes d'hygiène, de santé, de constructions très précaires, etc. Mon passage en analyse m'avait donc permis de me rendre compte de mes « angles morts » qui étaient justement cachés par ce que je cherchais à démontrer. En psychanalyse, ce phénomène s'appelle le fantasme. Dans des échanges de mail avec Danielle Bastien, psychanalyste, celle-ci m'expliquait :

«Selon Freud incroyablement perspicace, nous ne pouvons découvrir l'autre quel qu'il soit, conjoint, ami, extra-terrestre ou barbare au sens de ce qui nous est étranger, la réalité extérieure donc, qu'en fonction de notre filtre personnel, ce qu'il a nommé fantasme. Loin des vulgarisations érotico-stupides, le fantasme est une sorte de pair de lunettes qui ne nous permet pas de voir le ciel tel qu'il est mais filtré avec nos propres balises existentielles (qui se sont construites quand nous devenions sujet). Chacun tente de s'en sortir, bien ou mal avec ce cadre interne qui s'est fabriqué en dehors de sa conscience (on pourrait dire inconsciemment). De cette vision tronquée, si l'on suit Freud naissent les grandes histoires d'amour, ou de haine, les grands projets y compris théoriques...» (Bastien, conversation personnelle). 
Cet héritage familial avait donc un biais sur ce que je (ne) voyais (pas). Mais, d'une manière plus dérangeante encore, l'on peut être amené à se demander si ce biais n'avait pas une conséquence sur ce que mes interlocuteurs me racontaient. Si je ne voyais pas qu'il était difficile de vivre dans un camp, dans une logique que j'appellerai ici d'« optimiste aveuglé », la personne en face de moi agissait peut-être aussi de la sorte. De fait, je vois souvent que mes interlocuteurs me montrent leur réalité sous un angle optimiste, celui de prendre le verre à moitié plein plutôt qu'à moitié vide. De fait, il me semble qu'en plus d'être ce que je vois, c'est ce que je "diffuse » sur ces terrains : l'importance de voir le détail qui marche plutôt que la montagne de difficultés à côté de ce détail. Une personne qui voudrait lire les parcours de vie des réfugiés sous l'angle de leurs traumas sera peut-être plus encline à avoir des discussions de "pessimiste aveuglé ", ne voyant que ces aspects du terrain. Là encore, comment ce genre d'attente ne transforme-t-elle pas les discours des gens? Sans pour autant avancer que l' «optimiste aveuglé » ou le " pessimiste aveuglé » ne peuvent voir d'autres réalités (et c'est là que je ne rejoins pas entièrement la pensée postmoderne, voir infra).

Dans un autre registre, nous devons continuer à avoir conscience de notre propre subjectivité lorsque nous sommes en interaction sur le terrain. Toute la pensée " post-» a insisté là-dessus : les post-modernes, les féministes, les post-coloniaux, etc. Il faut inévitablement réfléchir à son rôle personnel lorsque nous sommes en interaction avec le terrain : que nous soyons une femme ou un homme dans une société particulière, un «Blanc» en Afrique, etc. De manière moins sociologique et plus individuelle, l'autoanalyse est un travail qu'il faut aussi opérer. Le social dont nous sommes témoins continue à se construire autour de nous, et nous sommes inévitablement impliqués dans celui-ci. Nos émotions peuvent être mises à rude épreuve. Nous vivons avec une certaine intensité des évènements particuliers qui font partie de la vie quotidienne de nos interlocuteurs, et de la nôtre le temps de. Sur mes terrains aux contextes explosifs, liés aux questions de guerre, de conflits cycliques, d'encampement, d'exil, etc., les évènements marquants et touchants mes émotions n'ont pas manqués ; marquant ainsi ma subjectivité en train de se faire.

11 Ainsi, par un hasard de calendrier, je me trouvais à Bukavu au printemps 2015 lorsque le président Burundais Nkurunziza a annoncé sa candidature pour un troisième mandat, ce qui a depuis replongé le pays dans une guerre étouffée $e^{8}$. Le lendemain de cette annonce, je me rendais en taxi à Bujumbura pour prendre mon avion. J'ai pris la route de la plaine de la Ruzizi, traversant Bujumbura pour aller rejoindre un ami chez lui. J'apprenais le soir, par un de mes contacts, que la plaine avait été traversée par des groupes rebelles se logeant au Congo et arrivant au Burundi suite à l'annonce. J'ai retrouvé Bujumbura le lendemain comme une ville morte, des barrages à gauche à droite, des pneus brûlés, quelques jeunes trainant dans des rues désertes sur des vélos. Et surtout, une peur se dégageait de chacune des parcelles derrières lesquelles les burundais se cachaient, dans leur maison. La ville entière "puait" la peur. C'était le calme avant une tempête inévitable, pour une population bien trop consciente de quels seraient les prochains épisodes. Le matin, alors que je traversais la plaine, les premiers morts étaient tombés9. Après avoir passé une journée sur le qui-vive, le cerveau concentré, en ébullition pour arriver à capter chacun des détails des scènes que je traversais au cas où, j'avais pu dégager la pression dans l'avion où j'étais en sécurité. Alors que j'avais l'impression de ne pas avoir ressenti la peur pendant la journée, des 
bêtes turbulences m'avaient alors parue terrifiantes, et il m'est resté de cet épisode une peur panique de chacun des avions que je prends.

En 2017, un nouvel épisode inattendu m'était arrivé sur un autre terrain, dans le camp de Kakuma au Nord du Kenya. Je passais mes journées avec un groupe de réfugiés congolais depuis un moment déjà. Mes mouvements ne ressemblaient pas à ceux d'un «Muzungu» (Blanc) normal, puisque je passais du temps avec eux, dans leurs lieux de sociabilité, sans faire aucun travail administratif - comme c'était habituellement le cas avec les travailleurs humanitaires. Un homme, auto-surnommé « Le Lion de Kakuma », avait l'habitude de passer des « deals» avec la police. Ici en vendant des faux certificats de réinstallation, là en coinçant des réfugiés dans des positions d'entre deux, à la limite de ce qu'ils pouvaient faire dans le camp. La peau blanche étant " comme un diamant " (pour reprendre le terme d'un de mes interlocuteurs), le Lion de Kakuma avait cru bon de se faire de l'argent sur mon dos. Profitant d'une position d'entre deux, d'une aiguille ayant dépassée un peu trop les 18 heures, à la lueur de la tombée du jour, il avait voulu me donner à la police de Kakuma, dont les exactions policières dans le camp constituaient purement et simplement des violences structurelles et en aucun cas des soucis de respect des règles. Sur le moment, je ne savais pas du tout de quoi il en retournait, mais mes interlocuteurs, le voyant venir, m'avaient mis sur une mototaxi avec laquelle j'avais pris la fuite. Je m'étais retrouvée, quelques rues plus loin, dans le logement (très précaire) de prostituées qui vivaient aux abords de l'aéroport mais dans la ville et non pas dans le camp. Par la même occasion, j'ai découvert leur réseau de prostitution duquel mes interlocuteurs vivaient. Cette nuit-là, un cambrioleur était venu voler les quelques objets des quelques chambrettes voisines de celle où je me trouvais (je ne sais par quel miracle le cambrioleur n'était pas venu dans la mienne). Les filles s'étaient rassemblées et mises à crier pour faire fuir le malotru. Un vol probablement organisé par quelqu'un qui a encore moins que les prostituées, ou, si l'on en croit les rumeurs de la région, par un Turkana, c'est-à-dire un kenyan de la région dont l'ethnie est considérée comme étant encore «traditionnelle ». À nouveau, je garde de cet épisode des souvenirs de peur, des traces d'acide dans mon ventre qui ne partiront pas. Ainsi peuvent se succéder les épisodes auxquels j'ai fait face, ici en me faisant coincée en plein milieu de la nuit par une bande de dix policiers armés, là en me retrouvant à dormir chez une famille dont l'homme m'annonçait être un criminel de guerre, ou encore en entendant depuis ma chambre d'hôtel trop reculée dans Goma des bruits de coup de feu réguliers et bien trop proches pour que je ne puisse jamais y fermer l'œil le temps de mon terrain.

13 Par ces exemples, j'aimerais affiner l'idée que toute situation vécue sur nos terrains est suffisante pour être "montée en analyse ». L'auto-analyse permet de tempérer les situations vécues. Restituer dans une monographie ces épisodes de manière brute, comme je les ai perçus et ressentis sur le moment, leurs donneraient plus d'importance qu'ils n'en ont dans un paysage social (et donc collectif) plus large que celui vécu par une seule subjectivité - la mienne. En théâtre, on appelle ça " être en carafe ", c'est-àdire ne plus être dans la justesse de la situation, mais dans une théâtralisation excessive $\mathrm{du}$ jeu d'acteur. Un retour réflexif sur la manière dont les épisodes de l'observation participante ont été vécus me semble donc nécessaire pour ne pas donner leurs plus d'importance que celle qu'ils ont réellement au regard d'une réalité sociologique ou politique particulière et plus large. Prenons l'exemple de l'homme qui m'annonçait être un criminel de guerre. Si je n'avais pas pris le temps d'analyser sa position ni ma réaction et mes émotions vécues, celles notamment qui sont à mettre en résonnance avec 
mon parcours de vie (une étudiante belge ayant grandi dans une société qui ne connait pas la guerre), alors je n'aurais pu évaluer sa position sociale à sa juste mesure. Je me souviens de mes postures de drama queen alors que j'expliquais à qui pouvait l'entendre que j'étais chamboulée d'avoir séjourné chez un «criminel de guerre ». Mais, dans la région des Grands Lacs, les personnes ayant participé à la guerre sont nombreuses. Donner une importance particulière à cet interlocuteur, aussi marquant eut-il été, ne permet pas de restituer sa position sociale dans un paysage plus large, et ne permet pas de replacer cet auteur à sa juste échelle, comme le rouage parmi tant d'autres d'un système politique et sociologique. L'idée est donc, de ne pas faire de cet interlocuteur un Laurent Nkunda (Seigneur de guerre de la région) en puissance, ou de faire des quelques coups de feu entendus la description de la prise de Goma en 2013 (c'est-à-dire un des moments les plus violents que la ville ait connue depuis le début de la guerre à $l^{\prime}$ Est du Congo $)^{10}$. Puisque nous continuons à vivre en étant sur nos terrains, la déconstruction des biais liés à nos propres subjectivités doit aussi s'opérer pour les épisodes que le terrain lui-même nous a donné à traverser.

\section{Le « second rôle » assigné à l'anthropologue}

Un des enjeux de l'observation participante est d'arriver à se faire une place sur le terrain. Pour cela, il faut avoir un statut social identifiable par nos interlocuteurs, pour mettre un pied dans leur vie quotidienne. Ainsi, il m'est arrivé d'avoir le statut de "professeur» lorsque je donnais des cours, ou celui de «reporter» ou de "photographe " lorsque je faisais, en parallèle, des petits reportages. Les statuts peuvent relever d'autres types de catégories, comme celui de "amie de " ou de " voisine de ». Toujours est-il que nous avons un rôle sur les terrains dans lesquels nous évoluons. Je me suis rendue compte qu'il y avait souvent un "second rôle" plus invisible qui m'était assigné, rôle dont je n'avais absolument pas conscience au début (et donc auquel je n'étais pas préparée), qu'il me semble nécessaire de comprendre avec les outils des psychologues ou des travailleurs sociaux. "Quelque chose", se rejouait chez certains interlocuteurs de terrain. Ils projetaient littéralement en moi une « image » ou un "rôle » qui, sans que je ne maittrise les outils des psychologues, pouvait relever de l'ordre du transfert. De manière récurrente, après mes terrains, je recevais des messages de mes interlocuteurs me disant de manière un peu trop soutenue que je leur manquais, voire qu'ils étaient tombés amoureux de moi. Certains en venaient même très étonnamment à réinventer leurs souvenir pour se rappeler que nous avions eu une histoire ensemble.

Alors que j'en parlais avec un collègue (homme) anthropologue, doctorant lui aussi, qui m'accompagnait sur un terrain, il me disait: Ah oui! Il parait que ça arrive tout le temps avec les anthropologues femmes! Leurs interlocuteurs tombent amoureux d'elles. Dans la littérature que j'ai parcourue, c'est pourtant un impensé (ou du moins un peu pensé) de la démarche anthropologique - voire un tabou qui relèverait de conversations de couloirs. Il existe bien une littérature sur les relations intimes et amoureuses sur les terrains, voire sur les anthropologues qui seraient «devenus natifs». On peut citer notamment l'article de Laurence Nicolas (2008) L'empathie: aporie ou doute méthodologique? De l'affection à la méthode, une anthropologue s'étant mariée sur son terrain et qui réfléchit à l'empathie à partir de cette posture particulière ${ }^{11}$. Mais, il existe moins une littérature sur les projections amoureuses des interlocuteurs envers 
nous, dues à notre présence d'acteur extérieur empathique, et surtout très attentif ${ }^{12}$ à leurs moindres faits et gestes. Que cela soit une projection amoureuse, ou une projection d'une autre sorte, c'est ce « second rôle » que je voudrais questionner ici.

Dans "Les Carnets de l'éducateur ", manuel à destination des (futurs) éducateurs, ce genre de phénomène est décrit.

«Sur le plan relationnel, l'éducateur se retrouve régulièrement, de manière délibérée ou non, dans des rôles assumés ou obligés, qui le dépassent peu ou prou. 'Sur le plan de l'imaginaire, la rencontre avec une personne réveille des images de projection et de refoulement de toutes sortes (attentes, transferts, rivalités...) qui colorent de façon régulière la rencontre présente » (Capul, Lemay, 1996 : p. 124).

Et donc comme pôle identificatoire et projectif, l'éducateur est amené à "jouer" plus ou moins longuement, en voyageant éventuellement de l'un à l'autre un rôle

« de contenant, de protection, d'accompagnateur, d'auxiliaire de moi, de substitut parental, de témoin de réalité, d'organisateur, de modificateur de comportement, d'intermédiaire, de révélateur de malaises individuels et sociaux. » (Davagle, 2012, p. 39)

Plus loin, le manuel explique que cet aspect est réglé, pensé, expliqué aux bénéficiaires, et ce, dans le cadre d'un mandat particulier, qui est celui posé par l'institution et qui relève de l'aide à la personne. Point d'aide à la personne, point de mandat, point d'institution partenaire pour l'anthropologue. Là où l'éducateur, également accompagnateur de quotidien, essaye d'expliquer clairement la distance qui le sépare de son interlocuteur, l'anthropologue, lui, fait tout pour effacer cette distance. Fait tout pour «Going Native », selon l'éthique philosophique qui lui est propre. Là où le travail de l'éducateur ou du psychologue est d'aider, le rôle de l'anthropologue est de comprendre, la variable «aide» étant a maxima politique, a minima un trait de personnalité dans des interactions quotidiennes, voire totalement inexistante.

C'est probablement une des raisons de l'impensé (ou du peu pensé) de ce genre de dynamiques sur nos terrains. Pourtant, lors de mon premier terrain, quelques jours après avoir "plongé » dans mon observation participante, un travailleur humanitaire m'avait fait la remarque qu'il n'était pas " éthique » d'établir des relations "proches " avec des réfugiés. J'avais à cette époque, «blindé» un argumentaire éthique et épistémologique qui justifiait le contraire. Je m'inscrivais dans la tradition méthodologique de la discipline qui se voulait, par le biais de l'observation participante, prendre ses interlocuteurs d'égal à égal. Pour citer l'un des nombreux auteurs défendant cette posture que je partageais, François Laplantine (2018 : pp. 20-21) avance :

« Je préconise de revenir à la sobriété et à la modestie de l'ethnographie c'est-à-dire de la recherche d'une co-construction du sens dans laquelle tous les acteurs sont à égalité. Dans cette dernière il n'y a pas de méta-langage, pas de méta-histoire, pas de méta-culture c'est-à-dire d'au-delà des cultures singulières. Il n'y a pas de position hiérarchique de surplomb car on ne remonte pas à l'un pas plus que l'on n'en descend. L'ethnographie nous conduit à remettre en question la dualité du signifiant et du signifié qui n'a rien d'universel et à abandonner la pensée étagée qui postule un dessus et un dessous, un dedans et un dehors, un centre et des périphéries et reconstruit le monde hiérarchiquement et hégémoniquement en distinguant et en subordonnant des régimes, des instances, des étages. »

19 Cet argumentaire de modestie, je l'ai tenu pendant longtemps, jusqu'à ce qu'un évènement tragique ne m'oblige à me repositionner. Je défendais alors dur comme fer l'éthique de l'observation participante qui permettait de considérer les réfugiés dans 
leur "humanité », de leur redonner une dignité face aux politiques de gestion des migrations par trop violentes (Vermylen, 2016). Je n'avais probablement pas compris que la remarque du travailleur humanitaire découlait d'une tradition du travail social, extrêmement conscient de ces dynamiques que nos outils littéraires et philosophiques ne permettaient pas de penser.

La première fois qu'un de mes interlocuteurs était tombé amoureux de moi, je me souviens m'être confiée à une de mes collègues de mon profond malaise. Il venait de me téléphoner. Un grand sourire s'entendait dans sa voix lorsqu'il m'annonçait qu'il avait failli dire en interview de réinstallation qu'il avait une fiancée en Europe, et que cette fiancée... c'était moi. Ma collègue m'avait alors répondu : Et toi ? Tu es aussi amoureuse? Est-ce que tu es mal à l'aise parce que toi aussi tu tombes amoureuse? Tu as l'impression que tu n'as pas le droit de tomber amoureuse de lui ?, qui m'avait alors semblé culpabilisant. Voilà les anthropologues - m'étais-je dit. Une bande de fous vivants dans des dénis d'inégalités. Dans des dénis de leur propre positionnalité. Je m'incluais bien évidemment dans la bande de fous. Puisque, contrairement à la question de ma collègue et bien que j'avais beaucoup d'estime pour lui, je n'étais pas tombée amoureuse de mon interlocuteur. Il ne représentait pas, pour moi, un univers de projections particulières qui pouvait m'amener à voir en lui tout ce qu'une projection amoureuse peut engendrer. Mais mon malaise, que je n'osais plus lui formuler après cette question, était celui-ci : Mais qu'ai-je fait? N'ai-je pas posé une petite bombe en venant faire ces terrains de la sorte? Comment je ne me suis pas rendue compte que j'étais tout sauf respectueuse en prétendant, selon une posture ethnographique "de modestie », qu'il n'y avait pas unfossé entre les réfugiés et moi?

Ce malaise n'a été que plus grand lorsque, quelques mois après mes premiers terrains, j'apprenais le décès de cet interlocuteur. Voici un extrait de mon carnet de terrain de l'été 2018, lorsque je recroisais pour la première fois son meilleur ami, que je n'avais plus vu à cause de la «crise " burundaise et qui avait été l'un des premiers à m'annoncer la nouvelle :

Nous parlons aussi de la mort de Mathis ${ }^{13}$, dont j'avais besoin de parler avec lui. Il me dit que Mathis était son ami d'enfance, depuis la maternelle. Ils s'appelaient tous les jours tous les jours. Ils ont fait l'école ensemble, puis ils ont bifurqué au moment des études. Alfonse a pris l'économie, Mathis a pris les scientifiques. La mère de Mathis est burundaise et son père est Rega. Son frère est encore à Bujumbura et ils se croisent encore. Mais depuis la mort de Mathis, sa mère n'ose plus regarder Alfonse dans les yeux, parce que quand elle voit Alfonse elle voit son fils. Parce que vraiment c'étaient les meilleurs amis. La vie en Angleterre [où il était réinstallé] était difficile et Alfonse et Mathis s'appelaient tous les jours. Ils n'avaient pas de secret l'un pour l'autre vraiment, donc il lui disait combien la vie en Angleterre était difficile. Il disait que au moins il pouvait envoyer de l'argent à sa petite maman et lui donner la belle vie quoi. Mais donc au moment où la guerre a commencé au Burundi, Alphonse n'a plus eu de nouvelles pendant une semaine. Alors qu'ils s'étaient au téléphone tous les jours. Là il a appelé, et il a engueulé Mathis au téléphone quand il a décroché. Mais ce n'était pas Mathis, c'était son colocataire. Qui lui disait que Mathis était mort. Alphonse a perdu le contact du colocataire, ce qu'il semble regretter. Ils ont fait une autopsie et ils ont trouvé dans le corps du poison. C'est la famille qui l'a dit à Alphonse. Le corps a été enterré en UK. Quand je lui dis que les réfugiés ont fait une cagnotte pour pouvoir l'aider dans les frais de l'enterrement, il dit qu'il croit que cet argent n'est pas parvenu au bon 
endroit. Je lui dis que les réfugiés racontent qu'il a eu une maladie foudroyante, mais bon ce n'est pas ça. Il dit qu'ils ne savent pas ce qui s'est passé, et que c'est probablement mieux pour préserver son image. [Sans jamais mentionner le mot dans aucune de nos conversations à ce sujet, il s'agit donc bien d'un suicide.] Alfonse me dit, le regard à la fois fuyant et complice, gêné et empathique, fataliste et triste : « Tu le sais. Que Mathis était amoureux de toi. » (Extrait de carnet de terrain, Bukavu, 29 août 2018) qu'il s'agissait d'un suicide), mon premier réflexe avait été - je ne sais pas pourquoi d'aller voir son mur Facebook. Il ponctuait son dernier post d'un simple «A », initiale cachée mais dévoilée à qui pourrait le voir de mon prénom. La bombe posée m'envoyait à son tour ses projectiles, comme un juste retour à l'envoyeur, et cela faisait mal. J'ai maladivement cherché à savoir, avec confirmation à l'appui ${ }^{14}$, si ce " $\mathrm{A}$ " posté sur son mur Facebook quelques jours avant sa mort me concernait. Je n'arrêtais pas de me dire que j'aurais dû être plus attentive à ses appels incessants, réclamant que j'aille lui rendre visite en Angleterre où il avait été réinstallé (ce que, par ailleurs, je comptais faire dans le cadre de ma recherche). Que les énormes écarts d'attention que je lui portais entre mes moments "sur le terrain » et les moments «dans ma propre vie" étaient injustes. Je voulais remonter le temps et au moins pouvoir lui dire au revoir, qu'il avait, certes, eu une vie injuste, mais qu'il était une belle personne. Qu'il ne s'envole pas sans que je n'aie pu lui formuler cette pensée...

oilà que, une seconde fois, mon comportement engagé suivant mes convictions anthropologiques et politiques, qui me semblait être le plus éthique (et que j'avais défendu à la conférence des 30 ans de Migrinter avec des salves d'applaudissements de la part de mes pairs), n'était peut-être pas tout à fait éthique. La prise de conscience des ponts, des grands écarts, des fossés presque maximaux entre mes interlocuteurs et moi, rendait visible les dynamiques récurrentes de projections (de transferts ?). Car, à côté de l'histoire tragique de Mathis qui m'a fait réfléchir aux dynamiques d'inégalité entre lui et moi, ces projections, ces "seconds rôles" étaient récurrents sur mes terrains. Depuis, avec les maigres outils dont je dispose (c'est-à-dire mon bon sens), je tente de casser ces projections avec le plus de douceur possible, parfois maladroitement. J'essaye d'expliciter en détail mon rôle, ma position, non pas politique ( «je suis anthropologue »), mais au sein de la relation individuelle tissée sur le moment ( « n'oublie pas que la complicité que l'on a existe au sein d'un cadre dans lequel j'ai une position particulière »).

Ce n'est pas pour autant, qu'en dehors de toute dynamique de projections où mes interlocuteurs s'attachent à moi de façon fantasmée, moi je ne m'attache pas à mes interlocuteurs. Sans le savoir, je n'avais jamais pu me remettre de la mort de mon interlocuteur ${ }^{15}$. Puisque ce n'était pas tout à fait «ma vie à moi ", je n'avais pas pu participer au processus de deuil et je n'avais personne dans mon quotidien pour comprendre ou partager cette douleur avec moi. Ces questions de deuils impossibles des anthropologues ont été abordées au sein du Laboratoire d'Anthropologie Prospective lors de nos séminaires internes. Jacinthe Mazzocchetti faisait notamment référence aux grands écarts entre nos terrains et nos vies, difficiles à gérer au moment des deuils puisque notre entourage «ne comprend pas » notre perte. Bernard Charlier (2018) a également coordonné un colloque international intitulé «Les anthropologues et leurs morts " suite à la perte de ses deux interlocuteurs principaux ${ }^{16}$. Pour ma part,

e-Migrinter, $18 \mid 2019$ 
je jonglais entre des moments "dans ma vie» et des moments "dans la vie de mes terrains ", deux vies qui étaient parfois à mille lieues de correspondre l'une à l'autre, ce qui m'a empêché pendant un moment de faire un deuil serein. La réponse est récemment venue de mon terrain lui-même. Pour des raisons de recherche, je me suis rendue en avril 2018 au Canada, chez un de ses amis réinstallé là-bas avec sa famille. Là, j'ai pu déposer mes larmes sereinement, et échanger des souvenirs de lui. Je me sentais liée intimement à mon terrain, mais je n'avais plus vraiment envie de le brandir haut et fort comme argument politique pour convaincre quelque extrême droite de «l'humanité ${ }^{17}$ » de nos interlocuteurs. Là, je me suis rendue compte que, même si je ne comptais pas « devenir native », mes terrains faisaient partie de moi.

\section{L'absorption des « ambiances »}

Ma position individuelle sur mes terrains a donc été, à deux égards, productrice de biais que seule l'auto-analyse m'a permis de contourner. Dans une logique de contre-transfert, mes «angles morts » m'ont empêchée a) d'appréhender les camps dans leur aspects difficiles et $b$ ) de resituer les réalités particulières auxquelles j'étais confrontée dans des réalités sociologiques plus larges que celles concernant mon propre vécu. Réalisant qu'inconsciemment je jouais un "second rôle » auprès de certains interlocuteurs, dans une dynamique qui pourrait relever du transfert, j'ai repensé les fossés sociaux et les inégalités entre les réfugiés des camps et moi, et essayé de me repositionner autrement face à mes interlocuteurs. Dans ce point-ci, je reviens sur l'apport d'une auto-analyse de mes émotions, non plus pour parer les biais de mes analyses, mais pour apporter une complexité à celles-ci, comme une véritable donnée en soi ${ }^{18}$. C'est en cela que je dépasse la posture postmoderne qui stipule que sur nos terrains, on ne voit que ce qui nous concerne ; ou que l'on ne voit que ce que l'on veut voir. Si la subjectivité du chercheur est incontestable, il n'en reste pas moins que nous soyons témoins d'une réalité singulière, dont il est possible de témoigner; non pas malgré notre subjectivité, mais grâce à notre subjectivité.

Dans un très bel article qui décrit comment le travail ethnographique peut être comparé à celui des improvisateurs de jazz, Liisa Malkki revient sur le débat mené au sein de la discipline face au positivisme, aux sciences quantitatives et à l'accusation faite à l'anthropologie de n'être que subjective. Pour elle, on se trompe en ne pensant l'observation participante que par le biais de l'impossibilité d'objectiver la réalité (critique moins souvent formulée à l'égard des sciences quantitatives). Elle y répond en disant que puisque nous sommes au fait de notre subjectivité, nous pouvons l'éviter. Un échantillonnage sociologique d'une population «prise au hasard» ne permettrait pas de pointer exactement les faits sociaux qui font sens et donc qui font société ; et il serait, lui aussi, le reflet du "faire sens" du chercheur. Ce qui permettrait à l'ethnographe de rendre la réalité observée objectivable serait, justement, sa sensibilité. Pour elle,

"Anthropology is not a social science tout court, but something else. What that something else is has been notoriously difficult to name, precisely because it involves less a subject matter - which, after all, overlaps with that of other disciplines in the humanities and social sciences - than sensibility. This sensibility is similar to Bourdieu's (2000) concept of professional 'disposition'. [...] They are many thing that still (remarkably perhaps, given our degrees of specialization), go without saying among sociocultural anthropologist understanding that are widely shared. » (Malkki, 2007, pp. 162-163) 
preuve, selon elle, que l'on ne peut pas nous accuser de ne parler que de nos propres «fantasmes", pour reprendre le terme de Freud, est notre capacité à nous faire surprendre par le terrain. Les termes utilisés pour décrire ce phénomène ont été multiples dans la discipline. Au Laboratoire d'Anthropologie Prospective (LAAP-UCL), nous utilisons le terme de "basculer »; et j'ai moi-même développé le concept de « nœuds de terrain » (Vermylen, 2019). Tout d'un coup, le tableau général de l'ensemble des connexions, des relations, des compréhensions de différentes sphères de l'intime ne fonctionne plus selon nos schèmes de pensée à nous. Cela ne fait plus «cqfd » et l'harmonie est perdue. Il nous faut donc les relire au travers d'un nouveau prisme, un Autre prisme. Pour Jacinthe Mazzocchetti (entretien dans ce numéro) "basculer» signifie, « au travers d'une expérience de décentrement, de rencontres et de relations, à la fois le bouleversement qui résulte de la transformation de soi inhérente à l'ethnographie et la capacité progressive de comprendre le monde depuis les schèmes de pensées et les référents d'un autre ». François Laplantine (2018, p. 97) fait, lui, référence aux transformations que la culture est susceptible de provoquer :

« ce processus s'effectue à travers une période très longue et très lente de latence et de gestation - une trentaine d'années en ce qui me concerne -, un mouvement souterrain de maturation qui ne va pas sans crises et conflits [...]; la dissolution progressive des stéréotypes de l'étranger dans l'expérience singulière de l'étrangeté. ».

En plus de nier l'apport de la subjectivité, l'avènement des Lumières, du positivisme, et de toute neutralité scientifique aurait donc rendu illégitime le fait de «se faire influencer » pour faire science ${ }^{19}$. Et c'est ce « se faire influencer » que j'aimerais mettre en avant, au regard de mes expériences de terrain et de l'outil de l'auto-analyse comme méthode anthropologique. J'ai, au cours de ma démarche de thèse, décidé d'écouter mes propres émotions lorsque j'étais sur le terrain (ou dans la lignée de mes terrains) pour comprendre certaines des réalités de mes interlocuteurs. Non pas dans une logique d'empathie directe du style «tu me racontes que tu as vécu ça, donc je le ressens et le comprends moi aussi » - logique à laquelle l'on adhère ou non. Mais dans une logique d'écoute de mes émotions comme une actrice ayant évolué dans des écosystèmes sociaux particuliers le temps de mes terrains; ayant "absorbé » des « ambiances » (Laplantine, 2018) particulières.

Reprenons la notions d'«ambiance » développée par François Laplantine (2018: pp. 57-73). Pour lui, une ambiance est la globalité de ce qui se produit dans un lieu commun (collectif et partagé donc) et dans un espace-temps qui crée du lien. Une forme de «bulle », forcément collective, jamais individuelle. Elle précède le langage et est rebelle à la verbalisation; elle n'est jamais la somme des parties que l'on peut lister pour la décrire, mais elle est plus que cela.

«Elle est comme une scène dans laquelle nous nous trouvons, ce qui suppose un partage du sensible. Elle provoque un ensemble de sensations qui ne sont pas vécues isolément mais ensemble sans qu'il y ait nécessairement uniformité mais une temporalité qui est celle de l'unisson.» (p.59).

«La théorie des ambiances [...] ne se réfère pas à un sujet. Ce dernier y participe évidemment. Il n'est nullement aboli mais ne peut plus être considéré comme antécédent, prééminent et déterminant. Pour dire les choses autrement, je n'ai nullement l'initiative d'une ambiance. Je suis pris dans une ambiance qui peut me toucher, me troubler ou m'indisposer. » (p. 62-63).

«Nous ne sommes pas [...] avec une ambiance dans un rapport de choséité mais de transformation de la subjectivité. Pour analyser une ambiance, il faut d'abord 
l'éprouver, c'est-à-dire suspendre les relations oppositionnelles du sujet et de

l'objet, de l'intériorité et de l'extériorité. » (p. 67-68). pour faire thèse. Ce petit « quelque chose en plus » qui fait que nous sommes traversés par nos réalités de terrain, que nous sommes « construits " par nos réalités de terrain ${ }^{20}$. Encore faut-il être capable de repérer et de faire la part des choses entre les émotions qui nous concernent et celles qui concernent nos terrains. J'essaye donc, de faire régulièrement le point et de comprendre « ce qui m'appartient » et «ce qui appartient à ce que je vais décrire " - démarche qui probablement s'estompera au fil de mes années de terrain, puisque la distinction entre les deux sera de plus en plus mince. Suivant une démarche d'écoute de mes émotions, je suis dans une logique opposées à celle - tout aussi intéressante - défendue par d'autres auteurs et qui consiste à postuler que le vécu d'une émotion similaire autre part rend capable de comprendre une réalité sociale que l'on aurait été incapable de comprendre par ailleurs. Ainsi, Renato Rosaldo (1989), travaillant sur une société de coupeurs de tête, dit que ce n'est que lorsqu'il vécut le décès d'un de ses proches qu'il a pu comprendre la colère vive nécessaire à cette action. En écoutant sa propre émotion au moment de son deuil, cela a fait «tilt ». Il a donc, dans son vécu personnel, été traversé par une colère vive, qui a pu faire écho à celle de coupeurs de têtes. Mais il ne dit pas avoir été " contaminé " par la colère des coupeurs de tête eux-mêmes, tout comme il ne dit pas l'avoir comprise du fait d'avoir évolué dans leur environnement social pendant un temps. Ici, je suis donc bien dans une démarche inverse à la sienne. Je comprends des émotions qui existent dans les Grands Lacs du fait de mon interaction avec la région, et non pas du fait de mon parcours de vie personnel.

31 Kobelinsky (2010), dans son ouvrage L'accueil des demandeurs d'asile. Une ethnographie de l'attente, use de cette technique pour comprendre des dynamiques propres aux centres d'accueils des demandeurs d'asile. Dans ce texte, elle décrit comment, sur le terrain, elle se met à envier ses collègues ethnographes qui vivent des aventures incroyables. Elle aussi voudrait "faire des choses ", "se mettre en mouvement ", mais rien ne se passe autour d'elle. Elle s'ennuie profondément. Elle trouve le temps long, et celui-ci lui pèse. Dans un de ces moments de « tilt » comme les terrains peuvent nous en apporter, elle comprend que ce n'est pas elle qui s'ennuie, mais que tout le monde autour d'elle s'ennuie. En découle une très belle ethnographie de l'attente, où elle compare notamment les demandeurs d'asile non à Ulysse comme c'est souvent le cas, mais à Pénélope qui, tous les soirs, détisse la tapisserie qu'elle est en train de tisser. Surtout, elle dépeint avec justesse la violence du système de l'attente du statut de réfugié, décrivant des anémies, des pertes d'envie, des heures interminables passées devant la télévision.

Pour ma part, les deux émotions «étrangères » qui m'ont submergée sur mes terrains sont une colère sourde et intense (plus intense que n'importe quelle colère que je n'ai jamais pu ressentir) et une profonde paranoïa (sentiment qui était jusqu'alors totalement extérieur à mes schèmes de fonctionnement). La région des Grands Lacs en Afrique est une région qui, historiquement et cycliquement, connait des violences extrêmes. Sans rentrer dans une tentative d'explication des successions de ces conflits, il est important de garder à l'esprit que les émotions qui y sont vécues sont corolaires des horreurs traversées. Dans ce cas-ci, l'outil méthodologique de l'absorption des ambiances permet aussi de se rendre compte que ces émotions particulières vécues appartiennent à un contexte. Pour donner un exemple de cette colère qui m'animait au 
Burundi, nous pouvons revenir sur l'étude (détonante) de Léandre Simbananiye (2005) sur les noms des Burundais.es. Ainsi, un nom comme il en existe beaucoup d'autres est «Baranyanka » qui veut dire littéralement « ils me haïssent ».

«L'instance nommante parle d'une histoire de haine qui est antérieure à la naissance du nommé. Celui-ci est l'occasion d'inscrire dans l'histoire familiale le climat social qui prévaut, par un message destiné à ceux-là mêmes qui nourrissent de mauvaises intentions pour qu'ils sachent que celles-ci ne sont pas ignorées, ce qui est aussi une façon de mettre en garde. La personne nommée est ainsi avertie de l'hostilité qui règne dans l'environnement d'accueil et il devra savoir où mettre le pied. » (pp. 172,173)

Une histoire de haine, marquée à l'indélébile dans le choix des noms donnés aux enfants, dans une société de rapport au voisinage marquée par des trahisons à la chaîne.

Pour ma part, je me suis surprise, en écrivant à quatre mains une chanson avec un de mes interlocuteurs congolais, de presque $\mathrm{y}$ " cracher mes tripes ", ma colère, plus que lui :

Tala biso na biso nanduse. On danse jusqu'à la fin, pas de douleur. Pas de couleuvre quand on danse dans la sueur.

Feti na feti, la folie, pas le vomi, la momie [ici compris comme " copine »] danse.

Mais le cadavre tu me séduis. Jte veux pour la nuit, mais pas pour toute la vie.

Maintenant on va dans le haut. Jsuis le chengue des chengue. [Enfant des rues] Pas dans la rue par terre.

Kadogo, kadogo! [Enfant soldat] Qu'est-ce que tu as fait de beau ?! Ta guerre, la guerre, nique ta mère!

Caporal, Général. Je me retire et je vous souhaite la galle.

Moi je n'ai pas la dalle. Vous mangez le Congo. Bon appétit.

Feti na feti che le zoba. Enfant soldat. Marche en zombie. Viens ma momie.

Jte tire une balle et on part au ciel. Pour visiter le Dieu de la terre et de la mère. Pas de la guerre. C'est biso na biso.

Washa moto. On n'a pas de contrôle.

Ipenoze Trouboze. C'est un cobra sous la garde.

Cobra, Cobra, [métaphore pour le Rwanda qui a la mainmise sur le Congo et qui est considéré comme étant responsable de la guerre] garde ton bras. Moi je rampe et je garde ma fierté comme Lumumba!

Quiconque se rend à Goma (chef-lieu du Nord Kivu) serait capable de capter l'émotion extrêmement sombre et dure qui s'en dégage. C'est cette émotion qui est reflétée dans la chanson.

Pour ce qui est de la paranoïa, j'ai mis plus longtemps à m'en rendre compte, puisque le principe de la paranoïa est de croire en des sentiments de persécution. Un peu comme quand on a mal digéré quelque chose et que l'on est capable de l'assigner à un aliment en particulier, ma paranoïa ressentie correspond à des émotions retrouvées au sein des communautés burundaises. De la compréhension de ce sentiment, découle un article sur les dynamiques d'insécurité et de guerre cyclique au Burundi ${ }^{21}$. Mais, et c'est là que je pense voir dans ma théorie sur l'absorption des ambiances quelques bases solides pour notre méthodologie, je me suis rendue compte que Liisa Malkki (2007) avait, elle aussi, ressenti de la paranoïa sur ses terrains dans les camps de réfugiés Burundais. Dans une démarche d'auto-analyse dévoilée en aval de sa monographie, elle met cela sur le compte de son séjour dans le camp :

"At other times in the course of fieldwork in that refugee camp, fear forced me to think hard about my own imagination and to try to distinguish between paranoia and reasonable caution. [C'est moi qui souligne.] Sitting alone at night with my affectionate tomcat, I 
sometimes felt my stomach tighten into knots as I imagined possible scenarios (mostly being kicked out of the camp before being able to complete my fieldwork, and of getting people into trouble with the authorities somehow simply by having sought their company). I had to work hard to sort out the possible scenarios from the likely scenarios, to trace the surface of my fear, and then to translate that into action and inaction. These night battle with my own imagination sharpened my senses, shaped my research strategies, taught me about the kinds of social performativity involved in being a "researcher" or "a student", and, most important, they raised continual ethical concerns about anonymity, confidentiality, informed consent, and the general safety of the people whit whom I worked and lived. In this case, I would not know how neatly to separate affect from thought. More, insisting on that neat compartmentalization would have been analytically counterproductive. [...] Yet, in the ethnographic monograph I wrote on the basis of the fieldwork in Tanzania (Malkki 1995) [avec des réfugiés hutu burundais], little remains of the centrality of affect and the senses in the process of my fieldwork. Whether it was fear or pleasure, boredom or comfort, pathos or humor, I tended to erase it from the final written product, perhaps appropriately. Another thing I self-consciously avoided was writing long, narrative descriptions of the landscape, people's houses, their dress and mannerisms, and the mood or the atmosphere of a particular moment. I did not feel entitled to describe in that way, from the evidence of my own senses. » (Malkki 2007, pp. 175-176)

À noter que Malkki a eu le réflexe inverse du mien, c'est-à-dire essayer de détacher le plus possible ses propres émotions de l'analyse anthropologique. Mais, ayant également séjourné dans des camps, je n'y ai jamais ressenti un sentiment de paranoïa, contrairement à mes séjours à Bujumbura, capitale du Burundi. Et si cette paranoïa, ressentie tant par Malkki que par moi, était un élément complémentaire pour comprendre avec subtilité des réalités quotidiennes au Burundi ?

\section{Conclusion : une ouverture sur nos catharsis}

37 J'aimerais conclure mon article par une réflexion sur nos catharsis. Powdermaker, arrivée sur son premier terrain, se posait comme question: "Why was I here, I asked myself repeatedly. There seemed to be no adequate reason: anthropology, curiosity, careere - all seemed totally unimportant. Why had I come? I began to think of all the events which had preceded my arrival here." (1966, p. 53) Je me suis posée la même question quand le terrain a été difficile pour moi ; mais plus encore, lors de l'assassinat de deux experts des Nations Unies, défenseurs des droits de l'homme, morts au Kasaï ${ }^{22}$. Je me suis dit, probablement naïvement : c'est injuste et dingue, ces deux experts, en choisissant une carrière, se sont dit que les enjeux géopolitiques de la région étaient intéressants, que les droits de l'homme étaient importants, ou peut-être que les questions liées à la guerre valaient de s'y arrêter. Mais, en Europe, l'on ne sait plus ce que veut dire le mot "guerre " ${ }^{23}$. Les réalités de nos terrains en Afrique des Grands Lacs nous renvoient à ce constat que les différentes constitutions des droits de l'homme ${ }^{24}$ sont un rempart rudement efficace en Europe, une idée commune presque indépassable, une vraie protection contre la guerre dans le vrai sens du terme. Ces droits de l'homme, qui font partie intégrante de notre habitus d'Européen, nous donnent l'impression inconsciente d'avoir le même degré de protection lorsque nous sommes sur le terrain. Nous, Européens, ne savons pas ce que le danger de la guerre signifie. Mais sur nos terrains, le fameux concept des "Droits de l'Homme» ne consiste qu'en une maigre feuille de papier insignifiante. Même l'eau magique qui protège les Maï-Maï ${ }^{25}$ des balles semble plus efficace. 
En apprenant la mort de ces experts ou en acceptant les « absorptions » qui avaient été les miennes, je me suis rendue compte de ce grand écart entre les livres et la pratique. Ce qui m'intéressait par effet de catharsis quand je lisais et les réalités des terrains en guerres qui m'ont affectée par ricochet, étaient des choses radicalement différentes. Une chose est de lire bien confortablement dans son lit un matin de dimanche pluvieux, une autre est de se confronter à la réalité. Une chose est d'être assise avec passion et arrogance dans un bar bruxellois à refaire le monde à coup de bières, une autre est de déambuler dans ledit monde. Et encore, je n'ai fait que côtoyer des gens ayant des parcours de vie liés à la guerre, je n'ai fait que me balader dans des régions en tension, avec la protection que peut apporter le statut de «Blanc ». Mais j'ai appris qu'il était philosophiquement impossible de comprendre l'horreur de la guerre, que derrière le mot "innommable», il y avait bien quelque chose qui relevait de l'ordre de l'incompréhension. Tant que moi - ici entendu comme une personne lambda - je n'ai pas vécu l'assassinat d'un de mes proches par exemple, tout ce que la personne qui l'a vécu pourra me raconter ne sera que des mots pour moi. Les seules émotions que je pourrai poser sur ces mots seront de l'ordre de la catharsis. Je me rappelle d'un moment particulièrement dur et brisant dans ma vie et je crois comprendre, au travers de cette émotion, ce que l'autre raconte. Fort heureusement pour notre santé mentale, nous ne comprenons pas beaucoup plus. Et le jour où, par "absorption", nous comprenons un millième de ce que cela peut être, nous nous disons qu'il est temps que l'on se barre de là !

\section{BIBLIOGRAPHIE}

Alloatti, Magali N. (2019) A multi-sited ethnography on culturla scenes and international migration : Reflecting on sites, links and para-ethnographers, e-Migrinter, $\mathrm{n}^{\circ} 18$

Cefaï, Daniel ; Amiraux, Valérie (2002) Les risques du métier. Engagements problématiques en sciences sociales. Partie 1, Culture \& Conflits, nº 4 [Réf. du 02/10/2016] [Disponible sur Internet].

Cerwonka, Allaine ; Malkki, Liisa (2007) Improvising Theory. Process and Temporality in Ethnographic Fieldwork, Chicago, The University of Chicago Press, 224 p. (Book).

Charlier, Bernard (dir.) (2018, 7 et 8 juin) Les anthropologues et leurs morts, colloque international, Louvain, Laboratoire d'anthropologie prospective ; UC Louvain [Disponible sur Internet].

Chiantaretto, Jean-François (dir.) (2002) Écriture de soi et narcissisme, Ramonville Saint Agne, ERES, 144 p. (Actualité de la psychanalyse).

Davagle, Michel (dir.) (2012) Les carnets de l'éducateur 2013. Exploration de la profession, Louvain-LaNeuve, Ciaco, 418 p.

Desille, Amandine ; Manaï, Bochra ; Vermylen, Aurore (2019) Post-colonialisme, hypersécurisation des frontières et ethnographie. Entretien avec Jacinthe Mazzocchetti, $e$-Migrinter, $\mathrm{n}^{\circ} 18$. 
Devereux, George (1967) From Anxiety to Method in the Behavioural Sciences, Mouton, The Hague, 396 p.

Jullien, François (2012) Les droits de l'homme sont-ils universels ?, YouTube, 04/04/2012, [consultée le 20 novembre 2017], 52 min 10 [Disponible sur Internet].

Kobelninsky, Carolina (2010) L'accueil des demandeurs d'asile. Une ethnographie de l'attente, Paris, Cygne, $110 \mathrm{p}$.

Laplantine, François (2018) Penser le sensible, Barcelone, Agora, 223 p. (Pocket).

Le Texier, Thibault (2018) Histoire d'un mensonge, Paris, La découverte, 200 p. (Zone).

Malkki, Liisa (1995) Purity in Exile: Violence, Memory, and National Cosmology among Hutu Regugees in Tanzania, Chicago, University of Chicago Press, $352 \mathrm{p}$.

Mazzocchetti, Jacinthe (dir.) (2007) Le travail social de proximité en questions. Entre utopie et sombre conscience, Louvain-la-Neuve, Academia-Bruylant, 204 p.

Nicolas Laurence (2008) L'empathie, aporie ou doute méthodologique ?, Journal des anthropologues [Réf. du 08/02/2019] [Disponible sur Internet].

Ouattara, Fatoumata (2004) Une étrange familiarité. Les exigences de l'anthropologie du 'chez soi', Cahier d'études africaines, n¹75 [Réf. du 17/05/2018] [Disponible sur Internet].

Powdermaker, Hortense (1966) Stranger and Friend, the Way of an Anthropologist, New York, W. W. Norton, 315 p.

Pestre, Dominique (2003) Sciences, argent et politique : Un essai d'interprétation, Paris, Sciences en questions-IRNA, 202 p. (Quae gie).

Rosaldo, Renato (1989) Culture \& Truth: The Remaking of Social Analysis, Boston, Beacon Press, 288 p.

Simbananiye, Léandre (2005) Les noms de personne au Burundi : un support du lien social, Anthropologie et Société, vol. 29, n¹, pp. 167-181.

Vermylen, Aurore (2016) Une anthropologue dans un camp de réfugiés. Comment faire ethnographie dans un contexte d'imposition discursive institutionnelle?, Parcours anthropologique, $\mathrm{n}^{\circ} 11$ [Disponible sur Internet].

Vermylen, Aurore, (à paraître/2019) Que faire lorsque notre envie du 'dire' ne correspond pas à notre constat du 'voir' ? in Laurent, P.-J. (dir.) Les processus d'écriture en Anthropologie, Paris, L'Harmattan (Collection d'Investigations d'anthropologie prospective).

Xanthakou, Margarita (1995) De la mémoire à la méthode : George Devereux, tel qu'en nousmêmes..., L'Homme, vol. 35, n¹34, pp. 179-190.

\section{NOTES}

1. Devereux affirme qu'il est possible d'esquisser une science objective du social (les «behavioral science »), à condition que l'on prenne en considération les effets de transfert et de contretransfert. Lui détermine les étapes du travail scientifique comme étant les suivantes: «A scientific behavioral science must start with the scrutiny of the complex matrix of meanings in which all its relevant data are embedded (Devereux 1957a), and with a specification of the means whereby the investigator gain access to, or else elicit, as many of these meanings as possible. [...] The second step consist in the study of the behavioral scientist's personal involvement with his material, and on the distortion of reality which these 'countertransference' reaction entail, since 
the greatest obstacle to the creation of a scientific behavioral science is the investigator's improperly-used emotional involvement with his material, which, in the final analysis, is himself and therefor inevitably arouses anxieties. The third step consist in an analysis of the nature and locus of the partition between subject and observer. The fourth and (provisionally) last step which can be taken in the present state of our knowledge is the acceptance and exploitation of the observer's subjectivity, and of the fact that his presence influence ('disturbs') the behavior of an electron. The behavioral scientist must learn to admit that he never observes the behavioral event which 'would have taken place' in his absence, nor hears an account identical with that which the same narrator would give to another person. Fortunately, the so-called 'disturbance' created by the observer's existence and activities when properly exploited, are the cornerstones of a scientific behavioral science, and not - as in currently believed - deplorable contretemps, best disposed of by hurriedly sweeping under the rug. " (Devereux 1967, p.6)

2. "La tendance dominante de l'épistémologie occidentale est de tout séparer en deux: le concept et le percept, la raison et l'émotion, le signifié et le signifiant, le sujet et l'objet, le sujet et le social, l'écrit et l'oral, le vrai et le faux, la réalité et la fiction qui serait du mensonge » (Laplantine, 2018, p. 21).

3. «Courte » en termes de psychanalyse. De mémoire, j'ai dû faire deux fois six mois.

4. Peut-être même que toute bonne thèse en anthropologie s'écrit en deux livres : l'un sur sa propre analyse et l'un sur l'analyse de ses observations participantes. Mon zèle n'est pas à la hauteur de ce perfectionnisme-là, mais c'est en tout cas quelque chose qui me semble être un fondement méthodologique vers lequel il faut tendre. Voir à ce propos par exemple Ouattara (2004) ou Chiantaretto (2002).

5. «Conduite en 1971 par le professeur Philip Zimbardo, l' «expérience de Stanford sur la prison » a vu vingt-deux étudiant volontaires jouer les rôles de gardiens et de prisonniers au sein d'une fausse prison installée dans l'université de Stanford. L'expérience devait durer deux semaines, mais elle fut arrêtée au bout de six jours, résume Zimbardo, car les "gardiens se montrèrent brutaux et souvent sadiques et les prisonniers, après une tentative de rébellion, dociles et accommodants, même si la moitié d'entre eux furent si perturbés psychologiquement qu'ils durent être libérés plus tôt que prévu. » (Le Texier, couverture) Pour un retour critique de l'expérience de Stanford: “'Fake science', retour critique d'expérience à Stanford »: https:// www.franceculture.fr/emissions/la-suite-dans-les-idees/la-suite-dans-les-idees-du-samedi-05mai-2018.

6. Oliver Hirschbiegel (2001).

7. La réinstallation est un programme mis en place par l'UNHCR pour envoyer les réfugiés qui vivent dans des situations d'« exil prolongé » dans des pays tiers occidentaux, comme les ÉtatsUnis ou le Canada. J'ai, dans le cadre de ma thèse, réalisé des terrains dans ces deux derniers pays auprès d'interlocuteurs congolais réinstallés.

8. Voir notamment la chronologie de un an de crise au Burundi, Jeune Afrique, le 22 avril 2016 : https://www.jeuneafrique.com/319851/politique/chronologie-an-de-crise-burundi/

9. Voir «Burundi : violente répression des manifestations contre un troisième mandat de Nkurunziza », Jeune Afrique le 27 avril 2015 : https://www.jeuneafrique.com/230169/politique/ burundi-violente-r-pression-de-manifestations-contre-un-3e-mandat-de-nkurunziza//

10. Dans mon cas, ma réaction est celle d'amplifier les évènements que je traverse. Mais l'inverse est vrai aussi, nombreuses sont les personnes travaillant sur des contextes en guerre et qui les dédramatisent (comme de nombreux interlocuteurs d'ailleurs). Une grenade qui explose dans son jardin? Une bande de policiers qui nous braque l'arme chargée en plein milieu de la nuit « juste pour rire " ? Une accumulation de photos de cadavres reçues sur WhatsApp? «Bha, ce n'est pas très grave. C'est normal quoi. » 
11. À noter que Laurence Nicolas, dans son article, fait également référence aux effets de contretransferts (en faisant référence à Devereux), ce que j'appelle dans le premier point mes « angles morts ») et aux limites interprétatives que ceux-ci nous imposent.

12. "Attentif » ayant la même étymologie que "attentionné », la posture que l'on a vis-à-vis de nos interlocuteurs peut ressembler à des postures affectives.

13. Les noms sont modifiés

14. Notamment lors de ma conversation avec Alfonse à Bukavu en août 2018, mais également lors d'une conversation avec un autre de ses amis proches, réinstallé au Canada, en avril 2018.

15. Et aussi, je ne me rendais pas compte que je changeais ma méthode de travail en fonction de celui-ci. Prise de panique que cela puisse arriver de nouveau, j'ai commencé à faire des terrains multi-situés, pour ne jamais être très longtemps quelque part et pour ne pas voir se rejouer d'évènements de la sorte. Il s'agit là, je crois, d'un processus de deuil géré par le déni.

16. Pour revoir les vidéos du colloque: https://uclouvain.be/fr/instituts-recherche/iacchos/ laap/les-anthropologues-et-leurs-morts.html.

17. Grand mot s'il en est puisque tout comportement est humain, du plus sadique au plus généreux.

18. Et bien sûr non pas comme donnée exclusive.

19. Et ce, corolairement au fait de ne pas se faire « aveugler » ou « influencer » par la croyance en un Dieu: l'avènement des Lumières étant corolaire à l'athéisassions (ou a minima l'«agnosticisassion») de la société française (Julliens 2012). Nous sommes d'ailleurs dans une époque où il est valorisé de "ne pas tomber dans le panneau ", d'où la prolifération de théories conspirationnistes, où des groupes de gens «ne tombent pas dans le panneau » ensemble.

20. Il me semble très étonnant de dire que nous ne pouvons voir les réalités que par le biais de ce qui nous constitue nous, puisque nous ne sommes que le produit des Autres, que ce soit biologiquement, psychologiquement ou sociologiquement. Ce sont les différents apports des Autres côtoyés qui font la singularité de ce que nous sommes.

21. L'article, pour le moment en processus de peer review, est co-écrit avec Julien Moriceau et s'intitule «Censures et insécurités sur les rives du Lac Tanganyika. Ethnographie des silences, non-dits et secrets au Burundi ».

22. À ce propos : http://congoresearchgroup.org/the-assassination-of-the-un-experts-what-dowe-know/?lang=fr. Voir également les révélations récentes sur leur assassinat, confiné dans les «Congo files» et dévoilées par Rfi, Le Monde, Foreign Policy, Süddeutsche Zeitung et la télévision suédoise. http://www.rfi.fr/afrique/20181127-congo-files-enquete-unpol-derangeonu-rdc-kasai-experts-meurtre

23. Voir à ce propos les écrits de Michel Serres sur la période unique de paix que l'Europe est en train de vivre.

24. Constitution européenne avec un préambule sur les droits de l'homme, Déclaration universelle des Droits de l'Homme et du Citoyen de 1948, etc.

25. Les Maï-Maï (littéralement « eau-eau ») sont des groupes rebelles présents à l'Est du Congo, dont une des particularités est de se recouvrir le corps d'une eau sacrée pour que les balles rebondissent sur leur poitrine. Si tant est que l'on retrouve des Maï-Maï morts par balle, on dit alors qu'ils n'étaient pas des « vrais ». 


\section{RÉSUMÉS}

Cet article met en évidence les apports de l'auto-analyse pour dépasser la dichotomie « raisonémotion ». Nous verrons en quoi nos émotions peuvent tout aussi bien être un frein qu'un apport à la compréhension des réalités sociales que nous traversons. Il a été rédigé en vue d'une analyse de phénomènes liés à la méthodologie et s'inscrit dans la lignée des réflexions méthodologiques et épistémologiques de l'ethnographie et plus généralement de l'anthropologie. Entre les lignes, et grâce à de longs exemples empiriques, l'article parle aussi de réalités sociologiques propres à mes terrains : les questions de la mise en camp de réfugiés ayant fui l'Est de la RDC, les guerres cycliques et non-cycliques de la région des Grands Lacs en Afrique. Mon analyse se base sur une observation multi-située, menée depuis 2014 dans la région des Grands Lacs en Afrique (Est du Congo, Burundi, Rwanda, Ouganda), mais également en Afrique de l'Est (Kenya) et en Amérique du Nord, où j'ai suivi des réfugiés congolais définitivement réinstallés via un programme des Nations Unies. Qu'elles soient réfugiées ou non, les personnes enquêtées ont vécu de près les réalités de la guerre.

\section{INDEX}

Index géographique : République Démocratique du Congo, Burundi, Rwanda, Ouganda, Kenya

Mots-clés : camps de réfugiés, guerres, relations enquêteurs-enquêtés, analyse critique, méthodologie

\section{AUTEUR}

\section{AURORE VERMYLEN}

PhD student, laboratoire d'anthropologie prospective (LAAP), UCL (Belgique) ; Bourse FRESH

(F.R.S.-FNRS)

aurore.vermylen@uclouvain.be 